\title{
An aneurysmal bone cyst ruptured and compressed the spinal cord: a case report
}

\author{
Yang Gu ${ }^{1 \#}$, Xin Chen ${ }^{2 \#}$, Linquan Zhou ${ }^{1 \#}$, Wenge Liu ${ }^{1}$ \\ ${ }^{1}$ Department of Orthopedic Surgery, Affiliated Union Hospital of Fujian Medical University, Fuzhou, China; ${ }^{2}$ Department of Orthopedic Surgery, \\ The First Affiliated Hospital of Xiamen University, Xiamen, China \\ \#These authors contributed equally to this work. \\ Correspondence to: Wenge Liu, MD, PhD. Department of Orthopedic Surgery, Affiliated Union Hospital of Fujian Medical University, 29 Xinquan \\ Road, Gulou District, Fuzhou 350001, China. Email: 13705977551@163.com.
}

\begin{abstract}
Spinal aneurysmal bone cyst $(\mathrm{ABC})$ is a rare benign bone lesion with various prognosis. Common clinical symptoms of spinal ABCs include local pain, swelling. But we presented a case of a teenager girl who exhibited symptoms of acute thoracic cord compression after a slight trauma and was then diagnosed with $\mathrm{ABC}$ in her thoracic spine. A unique aspect is that this patient did not have symptoms before she fell down on her hip, and had an acute worsening of her neurological deficits. In the vast majority of cases, for a teenager, the trauma on the spine is tiny after falling down on the hips. That is the reason we initially felt confused before she had an urgent CT scan. In order to achieve early decompression of the thoracic cord and stabilization of the local spine around T4, we proceeded with urgent lesion resection and pedicle screws fixation from T2 to T5 to remove the liquid containing cyst and achieve spinal stability. Postoperative pathology indicated the lesion was an ABC. The patient gained good neurological recovery without any adverse effect in the final follow-up. We believe spinal ABC of teenagers can have no symptoms until a slight trauma leading to acute neurological deficits. Careful preparing for emergency surgery, prompt resection of the lesion as well as spinal stability reconstruction can promote good recovery and minimal adverse effect.
\end{abstract}

Keywords: Thoracic cord; aneurysmal bone cyst (ABC); case report

Submitted Feb 20, 2020. Accepted for publication Jun 19, 2020.

doi: $10.21037 /$ tcr-20-1124

View this article at: http://dx.doi.org/10.21037/tcr-20-1124

\section{Introduction}

Jaffe and Lichenstein first described aneurysmal bone cyst $(\mathrm{ABC})$ as an expanding lesion consisting of blood-filled spaces of variable size separated by connective tissue septa with a low incidence rate, ranging from 0.14 to 0.32 per 100,000 individuals $(1,2)$. It most commonly occurs in individuals during their second decade of life. Females are slightly more affected with an estimated M:F sex ratio of $1: 1.16(3)$.

ABCs may affect any bone in the body, especially the metaphysis of long bones, including the femur, tibia/fibula, and upper extremity (4). Approximately $25 \%$ of these tumors arise from the spine. The distribution of spinal lesions is controversial. Some reports (5) showed that the lumbar spine was frequently involved, while other reports showed that the cervical and thoracic spine, especially thoracic spine, were more frequently involved $(6,7)$. Common clinical symptoms of spinal ABCs include local pain, swelling, muscle stiffness, and movement restriction; they are generally found a few months after symptom presentation (8). Neurologic deficit may happen with the infiltration and compression of nerve roots or spinal cord. However, some spinal ABCs do not have obvious symptoms until sudden causes happen, such as trauma, which may lead to pathological fracture.

Spinal ABCs are associated with anatomical challenges and increased hazards of neurological deficits, surgical morbidity and recurrence (9). Although these bone lesions are benign, they are locally aggressive and destructive and continue to expand until treated (8). Current treatment 
options include curettage, complete wide resection (with or without bone grafting) with stabilization of the spine. In order to reduce recurrence, many methods are used including cement, high-speed burr, phenol, and cryotherapy. Moreover, alternative strategies such as arterial embolization (either as an adjunct to surgery or as sole therapy), radiation therapy, sclerotherapy, percutaneous doxycycline, bisphosphonate medical therapy and denosumab are reported as the innovation of therapy (1). However, if patients with ABCs suffered from spinal cord compression or spinal instability, curettage and complete resection are more recommended.

Here, we will report a case of a teenage girl who exhibited symptoms of acute thoracic cord compression after a slight trauma and was then diagnosed with $\mathrm{ABC}$ in her thoracic spine. To our knowledge, it is the first report of spinal $\mathrm{ABC}$ without any clinical symptoms before a slight trauma and followed by acute neurologic compression. We present the following article in accordance with the CARE reporting checklist.

\section{Case presentation}

\section{History and clinical manifestations}

A 12-year-old girl from Xiamen, China fell down on her hip when playing at her school on September 19, 2018. She then presented with low back pain, numbness and weakness in both legs. Within several minutes, her left leg numbness alleviated, but numbness still existed in her right foot. On neurological examination, both legs' dorsiflexion weakness (motor power: left grade 3, right grade 2) and sensory impairment in $\mathrm{T} 5$ and below were observed from the patient. Furthermore, muscular tension of both legs was higher with right leg straight leg raising test $70^{\circ}$, positive tendon reflex and Babinski sign on both sides. She denied any history of similar symptoms and other disease. No family including relevant genetic information could be found. All procedures performed in studies involving human participants were in accordance with the ethical standards of the institutional research committee and with the Helsinki Declaration (as revised in 2013). The patient and her legal guardians were informed that data and figures from the case would be submitted for publication, and gave their consent.

\section{Imaging manifestations}

Plain X-ray, computed tomography (CT) scan and magnetic resonance imaging (MRI) of lumbar vertebra did not show any unusual signs. However, CT scan of thoracic vertebra revealed irregularly shaped lesions on the posterior elements of T3-4. MRI of thoracic vertebra certified the findings of the CT scan and also discovered these abnormal lesions were filled with some kind of liquid with fluid-fluid levels. Additionally, the main abnormal sites did not invade the surrounding muscles or ribs, but made the vertebral canal narrow and oppressed T3-4 level thoracic cord. Based on preoperative radiologic examinations, cystic benign tumor was the primary impression and emergency surgical treatment was considered to decompress the spinal cord (Figure 1).

\section{Treatment}

After preparation of emergency surgery, posterior thoracic spine laminectomy was performed during surgery to remove the liquid-containing cyst. The patient was positioned prone on a padded spinal operating frame under general anesthesia. A $3 \mathrm{~cm}$ long midline incision was made over the T3-4 area. After deepening the dissection in the midline using the electrocautery knife through the superficial fasciae and stripping erector spinae muscle, we reached the periosteum and then repeated the procedure until the desired number of vertebrae were exposed. No abnormal structure was discovered in fasciae or muscle during the exposure process. However, the spinous process, and the vertebral and bilateral zygapophyseal joints of T3 were invaded by the lesion, which looked like calcified sarcoid. The lesion expanded into the vertebral canal and also oppressed the spinal cord. The bilateral zygapophyseal joints of T4 were also involved.

We carefully used the ultrasonic osteotome to keep the lesion unbroken during the resection. The excision extension concluded the whole appendix of T3 and bilateral zygapophyseal joints of T4 to relieve spinal cord compression. After the lesionectomy, a large amount of bloody liquid outflowed from the lacerated fissure when the lesion wall was cut by a surgical assistant in order to observe the interior of the lesion. Moreover, we implanted eight pedicle screws from $\mathrm{T} 2$ to $\mathrm{T} 5$ in order to maintain the stability of the column (Figure 2). The total blood loss was $800 \mathrm{~mL}$ and the operation time was around 100 minutes.

\section{Postoperative course}

After the operation on September 20, 2018, the patient 

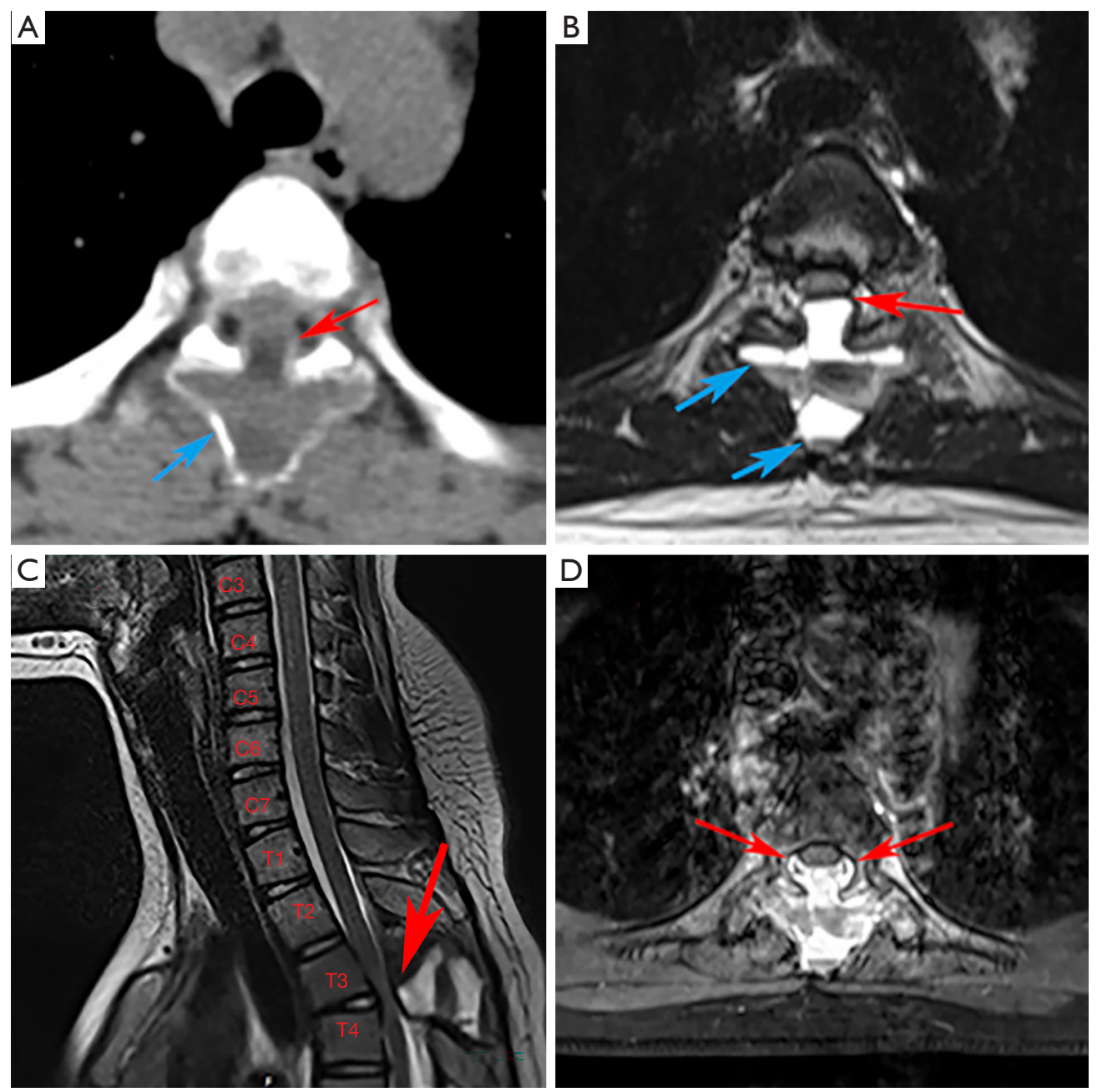

Figure 1 (A) Axial computed tomography scan: thoracic cord compression (red arrow) and osseous borders (blue arrow). (B) Axial magnetic resonance imaging: fluid-fluid levels (blue arrows) and compression (red arrow). (C) Sagittal magnetic resonance imaging: compression by the lesion. (D) Enhanced magnetic resonance imaging: both posterior and bilateral compression.

had a huge relief in her sensory impairment. There was no numbness in both of her legs and bilateral motor power enhanced instantly (left grade 4, right grade 4). No pathological reflection existed except Babinski sign of both sides.

Postoperative pathology indicated the T3 appendix lesion was an $\mathrm{ABC}$ with peripheral reactive bone formation as well as small amounts of dead bone formation.

Three weeks later on October 10, 2018, the patient gained a total sensory and motor recovery. She could walk around freely without any assistive walking devices. In addition, all pathological reflection disappeared. One year later on September 18, 2019, we had the follow-up CT scan and MRI to confirm no recurrence (Figure 3). The screws were exactly maintained in the pedicles and the canal was sufficiently enlarged.

\section{Discussion and conclusions}

$\mathrm{ABC}$ accounts for almost $1 \%$ of all bone tumors, with the prevalence at 0.14 to 0.32 per $100,000(3,5)$. It consists of blood-filled spaces separated by connective tissue septa containing bone trabeculation and osteoclastic giant cells (7).

Traditionally, ABC was regarded as arising due to increasing venous pressure leading to bone expansion. However, recently, primary $\mathrm{ABC}$ and secondary $\mathrm{ABC}$ are now identified as having different pathological origins. Although $\mathrm{ABC}$ is generally thought to have no malignance, 
it is believed that ubiquitin-specific protease 6 (USP6) gene mutation is involved in the formation of primary $\mathrm{ABC}$ by activating matrix metalloproteinase (MMP) to degrade extracellular matrix (ECM) components. In this way, ABC lesions can achieve bone growth and rapid expansion (10).

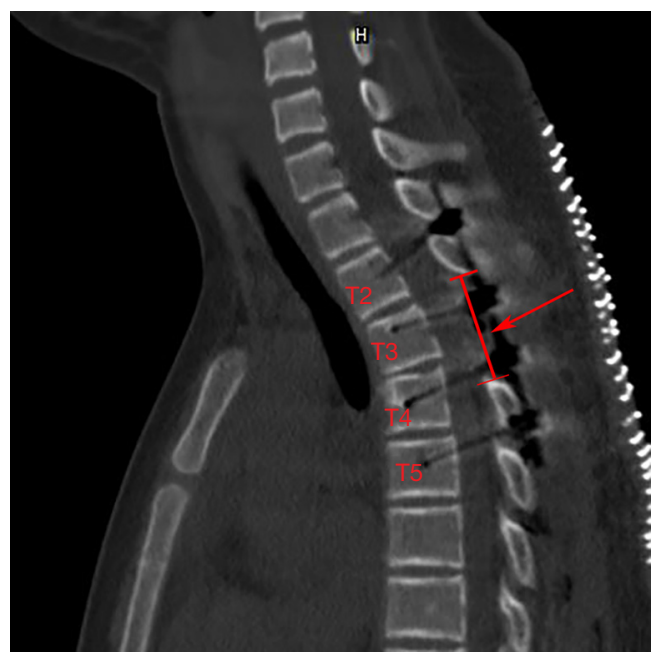

Figure 2 Computed tomography sagittal image after the surgery revealing a red arrow pointing at the length of the excised lamina and the implanted eight pedicle screws from T2 to T5 in order to maintain the stability of the column.
However, unlike primary $\mathrm{ABC}$, secondary $\mathrm{ABC}$, which occurs secondary to other bone tumors, presents no translocation or genetic aberrancy.

Just like the diagnosis of other bone tumors, three aspects including symptoms, radiographic studies and histological analysis should be considered by a doctor. Common clinical symptoms of spinal ABCs include local pain, swelling, muscle stiffness, and movement restriction. Some patients, like the girl in our case, do not have any symptoms before they are injured. None of these symptoms have the characteristics of specificity. In some cases, X-ray is often the first diagnosis clue of $\mathrm{ABC}$, usually appearing as a radiolucent cystic lesion circumscribed by a thin layer of the cortical bone. It looks like a balloon, and perhaps has septations in it that make it like a honeycomb or soap bubble. The most obvious differential diagnosis should be giant cell tumor of the bone. That is the reason plain graphs alone do not suffice in fully characterizing a soap bubblelike lesion. CT scan and MRI can define the osseous borders and fluid-fluid levels respectively. The fluid-fluid levels in MRI are due to blood of differing densities in the cyst atop one another among the interval septations. Sometimes, peri-lesional extension and edema can be observed in the tissue around the bone on MRI $(1,11)$. Since other lesions such as giant cell tumors, fracture through unicameral bone cysts or telangiectatic osteosarcoma (TOS) can

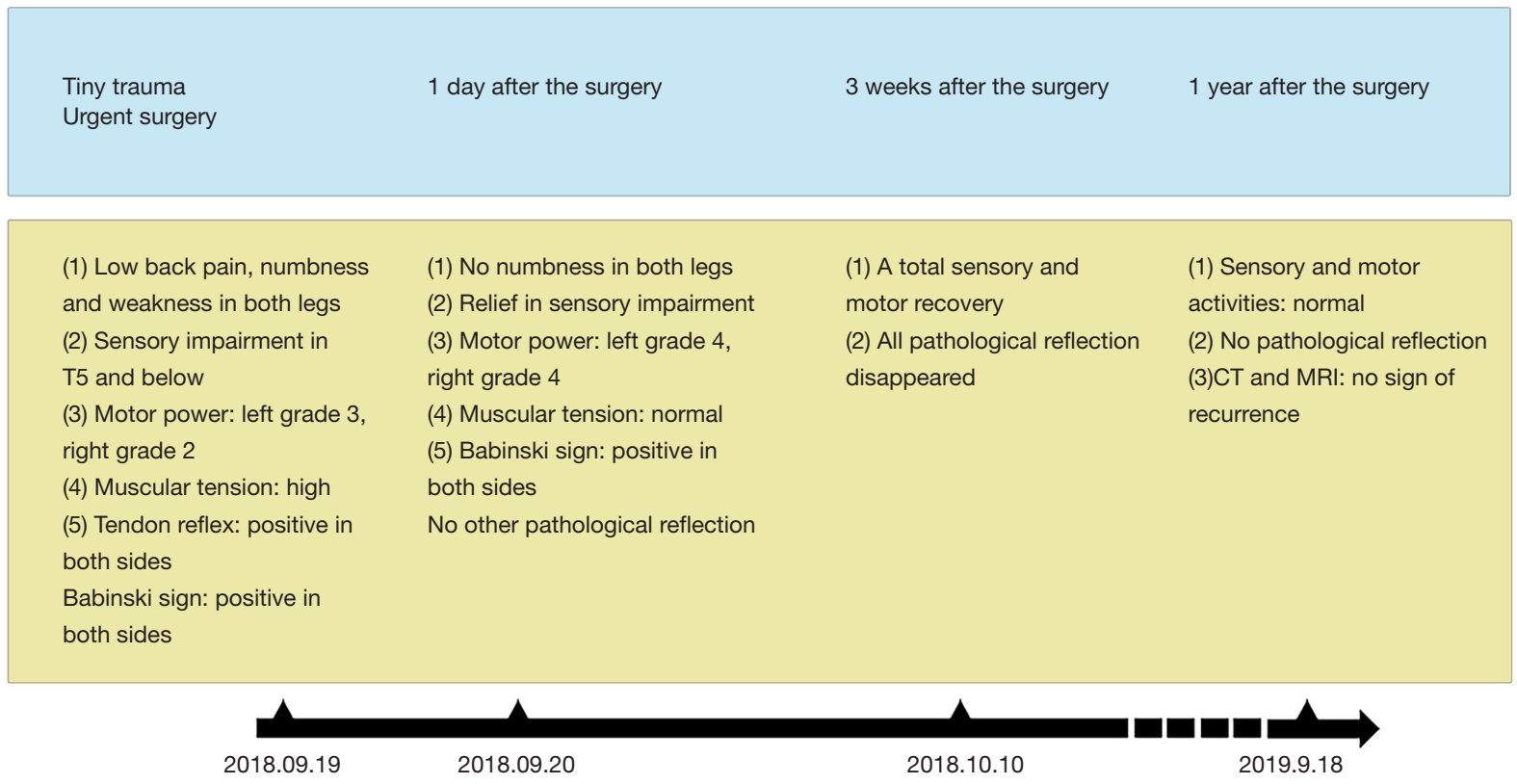

Figure 3 Treatment and follow-up timeline. 

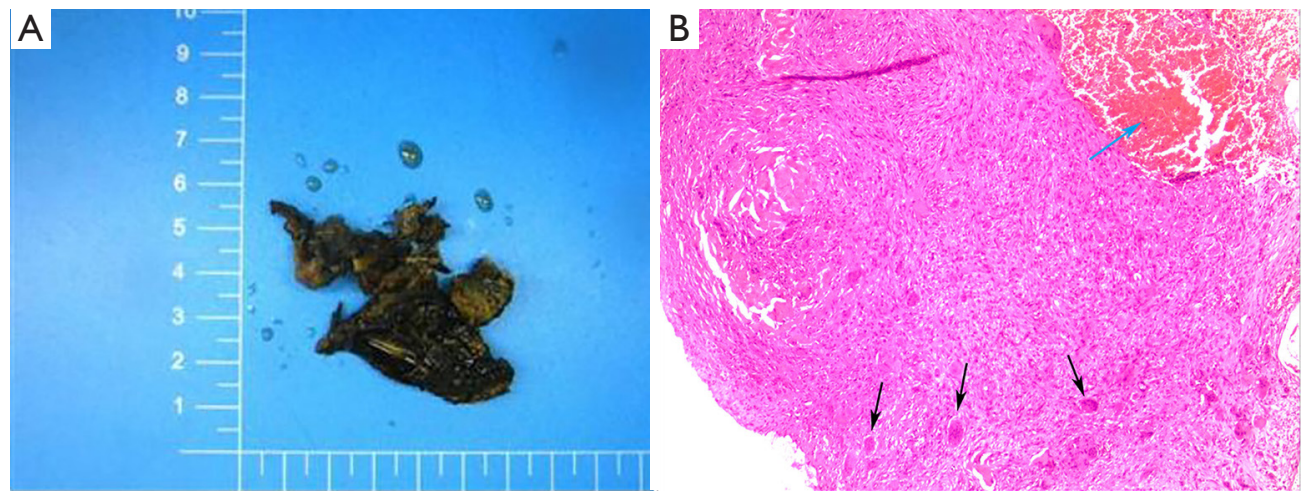

Figure 4 (A) The general view of the lesion with bloody liquid outflowed. (B) High power histology demonstrating scattered multinucleated giant cells (black arrows) within a dense background of bland fibroblasts in the septation and blood-filled cystic space (blue arrow); $\times 100$ times.

demonstrate these similar radiological features, it is clear that no one can diagnose $\mathrm{ABC}$ without histological analysis. Microscopic analysis reveals red blood cells and often pale brown hemosiderin with cyst-like spaces separated by solid portions containing osteoid, calcifications, fibroblasts and scattered giant cells (12) (Figure 4). The most important malignant lesion to differentiate from ABC is TOS, which exhibits malignant and sarcomatous cells in the interval septations as opposed to the septal proliferations of fibroblasts found in $\mathrm{ABC}$ (13). As a standard for diagnosis of $\mathrm{ABC}$, fine-needle incisional biopsy with histologic evaluation is currently done, but the risk of missed diagnosis is unacceptably high (14). The dilemma of missed diagnosis lies in the incisional biopsy and can be partly solved with the guidance of radiologic assistance. Otherwise, only samples from lesion resection can offer pathological details for the diagnosis of $\mathrm{ABC}$.

The special anatomical structure of the spine determines relatively higher treatment risks. The common treatment of choice for spinal $\mathrm{ABC}$, while controversial, may include curettage, embolization, radiation therapy, lension excision, or a combination of these (15). Moreover, many scholars proposed alternative management options. In several studies, Denosumab was reported to be effective in treating $\mathrm{ABC}$ with the mechanism of preventing activation and proliferation of osteoclasts by blocking the receptor activator of nuclear factor k-B ligand (RANKL), which results in tumor regression (16). Liu et al. reported the use of percutaneous albumin/doxycycline injection to treat $\mathrm{ABC}$ and compared it with open surgery, concluding that albumin/doxycycline injection was safe and effective in well-selected cases (17). Kieser et al. reported intravenous bisphosphonate therapy could be used as the definitive treatment for spinal ABC (18). Furthermore, surgical methods have become more minimally invasive. Shibuya reported the first case by using PELD (percutaneous endoscopic lumbar discectomy) to perform curettage with bone grafting (19). However, these alternative options can only be applied on well-selected patients without spinal cord compression or spinal instability.

In this case, a unique aspect is that our patient did not have symptoms before she fell down on her hip, and had an acute worsening of her neurological deficits. That is the reason we initially felt confused before she had an urgent CT scan. In the vast majority of cases, for a teenager, the trauma on the spine is tiny after falling down on the hips. The characteristic, but not unique, osseous borders on CT scan and fluid-fluid levels on MRI indicate the lesion may be an ABC. Moreover, the trauma might cause a pathological T4 fracture to narrow the vertebral canal and oppress the T3-4 level thoracic cord. A preoperative biopsy was not successful because of the urgent requirement of decompression. In order to achieve early decompression of the thoracic cord and stabilization of the local spine around T4, we proceeded with urgent surgery without preoperative embolization. To our best knowledge, there is only one previous study describing a 21-year-old pregnant woman who presented with progressive onset paraparesis with a lesion of thoracic ABC (20). Our case is the first description of acute thoracic compression after minor trauma associated with spinal ABC without any previous symptoms. It is still unclear how the minor trauma altered the lesion to compress the spinal cord since there were no previous symptoms. One possible explanation is the minor trauma 
led to a pathological fracture of the lesion. The patient's family had a limited amount of financial resources, so we also did not do further gene detection to check the USP6 mutation. Nonetheless, it is an effective and safe strategy to have urgent surgery, especially lesion resection to gain total decompression and stability, in a similar patient with acute neurological deficits from spinal $\mathrm{ABC}$.

Spinal ABC of teenagers can have no symptoms until a slight trauma leads to acute neurological deficits because of spinal cord compression. Although we have many options to treat spinal $\mathrm{ABC}$, they can only be applied in well-selected patients without spinal cord compression or spinal instability. Careful preparing for urgent surgery, prompt resection of the lesion as well as spinal stability reconstruction can promote good recovery and minimal adverse effect.

\section{Acknowledgments}

The authors would like to thank Qinchao $\mathrm{Wu}, \mathrm{MD}$ from Department of Pathology, The First Affiliated Hospital of Xiamen University for contributing histology slides to this article.

Funding: None.

\section{Footnote}

Conflicts of Interest: All authors have completed the ICMJE uniform disclosure form (available at http://dx.doi. org/10.21037/tcr-20-1124). The authors have no conflicts of interest to declare.

Ethical Statement: The authors are accountable for all aspects of the work in ensuring that questions related to the accuracy or integrity of any part of the work are appropriately investigated and resolved. All procedures performed in studies involving human participants were in accordance with the ethical standards of the institutional research committee and with the Helsinki Declaration (as revised in 2013). The patient and her legal guardians were informed that data and figures from the case would be submitted for publication, and gave their consent.

Open Access Statement: This is an Open Access article distributed in accordance with the Creative Commons Attribution-NonCommercial-NoDerivs 4.0 International License (CC BY-NC-ND 4.0), which permits the noncommercial replication and distribution of the article with the strict proviso that no changes or edits are made and the original work is properly cited (including links to both the formal publication through the relevant DOI and the license). See: https://creativecommons.org/licenses/by-nc-nd/4.0/.

\section{References}

1. Park HY, Yang SK, Sheppard WL, et al. Current management of aneurysmal bone cysts. Curr Rev Musculoskelet Med 2016;9:435-44.

2. Rapp TB, Ward JP, Alaia MJ. Aneurysmal bone cyst. J Am Acad Orthop Surg 2012;20:233-41.

3. Leithner A, Windhager R, Lang S, et al. Aneurysmal bone cyst. A population based epidemiologic study and literature review. Clin Orthop Relat Res 1999;(363):176-9.

4. Lopez LV, Rodriguez MG, Siegal GP, et al. Extraskeletal aneurysmal bone cyst: Report of a case and review of the literature. Pathol Res Pract 2017;213:1445-9.

5. Boriani S, De Iure F, Campanacci L, et al. Aneurysmal bone cyst of the mobile spine: report on 41 cases. Spine (Phila Pa 1976) 2001;26:27-35.

6. Papagelopoulos PJ, Currier BL, Shaughnessy WJ, et al. Aneurysmal bone cyst of the spine: management and outcome. Spine (Phila Pa 1976) 1998;23:621-8.

7. Aljoghaiman MS, Alhamad SM, Homan MA, et al. Aneurysmal bone cyst of the spine: Report of four cases and review of the literature. Interdiscip Neurosurg 2019;16:18-21.

8. Toorani B, Abdulla FA, Al Marzooq O. Challenges in the Treatment of Spinal Aneurysmal Bone Cysts. Bahrain Med Bull 2018;40:175-7.

9. Rosenblatt J, Koder A. Understanding Unicameral and Aneurysmal Bone Cysts. Pediatr Rev 2019;40:51-9.

10. Ye Y, Pringle LM, Lau AW, et al. TRE17/USP6 oncogene translocated in aneurysmal bone cyst induces matrix metalloproteinase production via activation of $\mathrm{NF}-\mathrm{\kappa B}$. Oncogene 2010;29:3619.

11. Mahnken A, Nolte-Ernsting C, Wildberger J, et al. Aneurysmal bone cyst: value of MR imaging and conventional radiography. Eur Radiol 2003;13:1118-24.

12. Kumar V, Abbas AK, Fausto N, et al. Robbins and Cotran pathologic basis of disease, professional edition e-book. Elsevier health sciences; 2014.

13. Murphey MD, wan Jaovisidha S, Temple HT, et al. Telangiectatic osteosarcoma: radiologic-pathologic comparison. Radiology 2003;229:545-53.

14. Creager AJ, Madden CR, Bergman S, et al. Aneurysmal bone cyst: fine-needle aspiration findings in 23 patients 
with clinical and radiologic correlation. Am J Clin Pathol 2007;128:740-5.

15. Zileli M, Isik HS, Ogut FE, et al. Aneurysmal bone cysts of the spine. Eur Spine J 2013;22:593-601.

16. Patel RS, Dhamne CA, Gopinathan A, et al. Denosumab: a potential treatment option for aneurysmal bone cyst of the atlas. Eur Spine J 2018;27:494-500.

17. Liu X, Han SB, Si G, et al. Percutaneous albumin/ doxycycline injection versus open surgery for aneurysmal bone cysts in the mobile spine. Eur Spine J 2019;28:1529-36.

Cite this article as: $\mathrm{Gu} \mathrm{Y,} \mathrm{Chen} \mathrm{X}$, Zhou L, Liu W. An aneurysmal bone cyst ruptured and compressed the spinal cord: a case report. Transl Cancer Res 2020;9(8):5008-5014. doi: $10.21037 /$ tcr-20-1124
18. Kieser DC, Mazas S, Cawley DT, et al. Bisphosphonate therapy for spinal aneurysmal bone cysts. Eur Spine J 2018;27:851-8.

19. Shibuya I, Dezawa A, Urayama S, et al. Surgical treatment of a lumbar aneurysmal bone cyst using percutaneous endoscopic lumbar discectomy. Eur Spine J 2018;27:368-74.

20. Li L, Tan LA, Wewel JT, et al. Spinal aneurysmal bone cyst presenting as acute paraparesis during pregnancy. J Clin Neurosci 2016;28:167-9. 